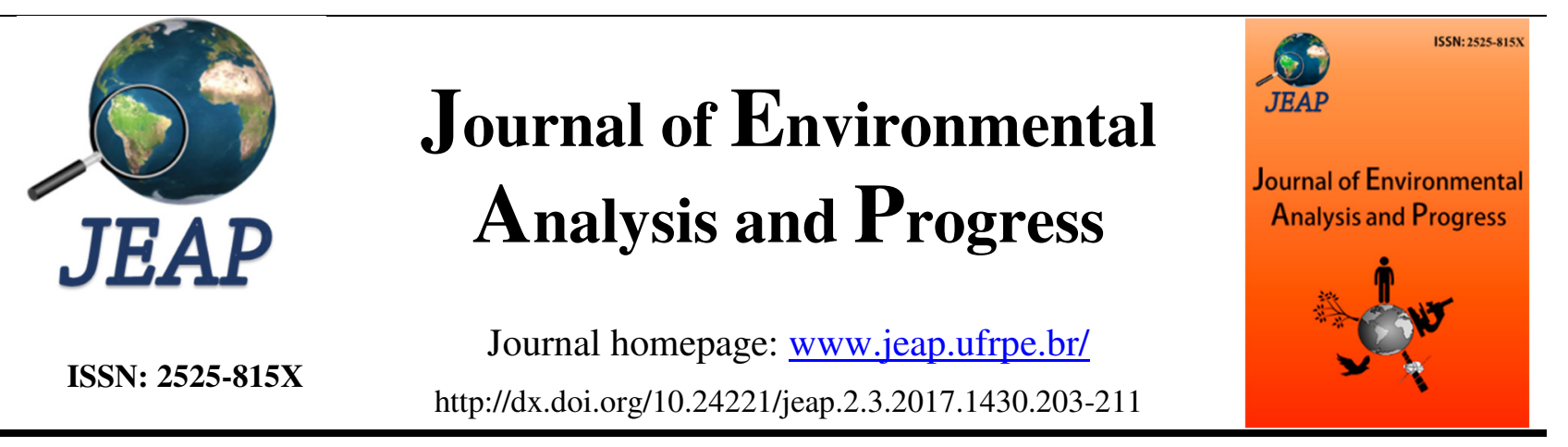

\title{
Estimativa da produção de sedimentos na bacia hidrográfica do submédio Rio São Francisco
}

\section{Estimate the sediment yield in the basin of lower-middle São Francisco River}

Madson Tavares Silva ${ }^{a}$, Pedro Vieira de Azevedo ${ }^{a}$, Vicente de Paulo Rodrigues da Silva ${ }^{a}$, Bruce Kelly da Nobrega Silva ${ }^{b}$, Everson Batista Mariano ${ }^{a}$, Marcos Renato Basílio Amorim ${ }^{a}$

a Universidade Federal de Campina Grande-UFCG, Unidade Acadêmica de Ciências Atmosféricas, Rua Aprígio Veloso, 882, Universitário, Campina Grande, Paraíba, Brasil. CEP: 58429-900. E-mail: madson.geotech@gmail.com; pvdeazevedo@gmail.com; vicente.paulo@ufcg.edu.br; eversonmariano@yahoo.com.br; marcosrenato85@gmail.com.

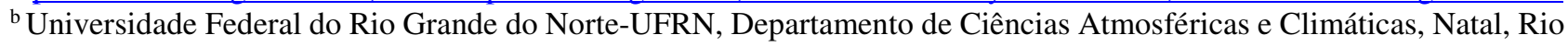
Grande do Norte, Brasil. CEP: 59078-970. E-mail: brucekellys@ hotmail.com

\begin{tabular}{|c|c|}
\hline A R T I C L E I N F O & A B S T R A C T \\
\hline Recebido 27 Jun 2017 & The mapping and assessment of risk of erosion is important for planning of natural \\
\hline Aceito 02 Jun 2017 & resource management tool. A considerable number of researchers have shown \\
\hline Publicado 31 Jun 2017 & interest in studies of environmental impacts caused by human activities that promote \\
\hline & $\begin{array}{l}\text { the accumulation of sediments in the river bed. The objective of this study was to } \\
\text { estimate the sediment yield from the Soil and Water Assessment Tool (SWAT) }\end{array}$ \\
\hline & model in the São Francisco river basin under the present condition of land use and \\
\hline & the meteorological stations of the National Institute of Meteorology (INMET) were \\
\hline & used. SWAT estimated soil erosion from the Universal Equation of Modified Soil \\
\hline & $\begin{array}{l}\text { Loss. The results of the simulations allowed the identification of areas with greater } \\
\text { potential for water erosion. Landscape management in watersheds through the }\end{array}$ \\
\hline & SWAT model can assist to identify areas susceptible to erosion processes. \\
\hline
\end{tabular}

Keywords: Hydrology modeling, surface runoff, SWAT.

\begin{abstract}
R E S U M O
O mapeamento e avaliação de risco de erosão é uma ferramenta importante para o planejamento da gestão de recursos naturais. Um considerável número de pesquisadores tem mostrado interesse por estudos de impactos ambientais causados por atividades humanas que promovem o acúmulo de sedimentos no leito dos rios. O objetivo deste estudo foi estimar a produção de sedimentos a partir do modelo Soil and Water Assessment Tool (SWAT) na bacia hidrográfica do submédio rio São Francisco na condição atual de uso e ocupação do solo. O período de simulação compreendeu os anos de 1993 a 2010 . Foram utilizados dados de entrada oriundos das estações meteorológicas do Instituto Nacional de Meteorologia (INMET). O SWAT estimou a erosão do solo a partir da Equação Universal de Perda de Solo Modificada. O resultado das simulações possibilitou a identificação de áreas com maior potencial à erosão hídrica. $\mathrm{O}$ gerenciamento da paisagem em bacias hidrográficas por meio do modelo SWAT pode ajudar na identificação de áreas susceptíveis aos processos de erosão.
\end{abstract}

Palavras-Chave: Modelagem hidrológica, escoamento superficial, SWAT.

\section{Introdução}

$\mathrm{O}$ interesse por estudos de impactos ambientais causados pelas atividades humanas tem movido um considerável número de pesquisadores a estudar novas ferramentas, inclusive aquelas que buscam simular as transformações ocorridas com 
as mudanças do clima (Silva et al., 2010; Sousa et al., 2010) e no uso da água e do solo (Oeurng et al., 2011).

Os modelos hidrológicos aplicados em bacias hidrográficas são cada vez mais usados para facilitar a gestão alternativa dos recursos hídricos e controle de inundações (Aragão et al., 2013; Pereira et al., 2014b; Fukunaga et al., 2015). Um dos modelos mais utilizados no mundo para simular os processos da erosão do solo e transporte de poluentes é o modelo hidrossedimentológico Soil and Water Assessment Tool (SWAT). Esse é um modelo distribuído que pode ser acoplado a um ambiente SIG e considera a variabilidade espacial das características físicas da bacia hidrográfica. Muitos estudos têm aplicado esse modelo para analisar os efeitos das mudanças no uso da terra sobre os fluxos hidrológicos em bacias hidrográficas (Setegn et al., 2010; Pereira et al., 2014a; Galharte et al., 2014, Silva et al., 2015; Silva et al., 2016a; Silva et al., 2016b). Entretanto, o SWAT pode ser inadequado para a simulação do escoamento em pequenas bacias (Kim \& Lee, 2010).

O entendimento de como as mudanças no uso do solo influenciam os padrões de variação das vazões ajudam a planejar estratégias que minimizem os efeitos indesejáveis de futuras mudanças no uso e ocupação do solo (CIBIN et al., 2010). Por outro lado, segundo Hundecha et al. (2008), há pouca informação científica sobre a relação quantitativa entre as propriedades do uso do solo e os mecanismos da geração de erosão e os processos inerentes ao transporte de sedimentos.
Assim, modelos matemáticos como o SWAT apresentam grande potencial para serem utilizados como instrumento de apoio ao planejamento de uso da terra, com o propósito de melhorar a qualidade do solo e da água de bacias hidrográficas. A hipótese básica deste estudo é que a produção de sedimentos pode ser aumentada pela mudança no uso de solo e, dessa forma, acelera o processo de degradação e desertificação da paisagem em bacias hidrográficas. Neste sentido, o estudo objetivou validar o modelo SWAT para simular o aporte de sedimentos na bacia hidrográfica do submédio rio São Francisco com base no cenário atual de uso do solo.

\section{Material e Métodos}

Área de estudo

O estudo foi desenvolvido na região do Submédio do Vale do rio São Francisco, que abrange áreas dos Estados da Bahia e Pernambuco, estendendo-se da cidade de Remanso até a cidade de Paulo Afonso, ambas no Estado da Bahia (Figura 1), com 110.446,00 km², ou 17\% da área da Bacia do rio São Francisco. Essa região tem 440 $\mathrm{km}$ de extensão e a sua população é de 1,944 milhões de habitantes. Ela está constituída pelas sub-bacias dos rios Pontal, Garças, Brígida, Pajeú, Moxotó e Xingó, pela margem esquerda; enquanto na margem direita ficam as sub-bacias de Tourão, Salgado, Vargem, Curaçá, Macuru e Poço Comprido, assim como descrito pela Companhia de Desenvolvimento dos Vales do São Francisco e do Parnaíba (CODEVASF, 1999).

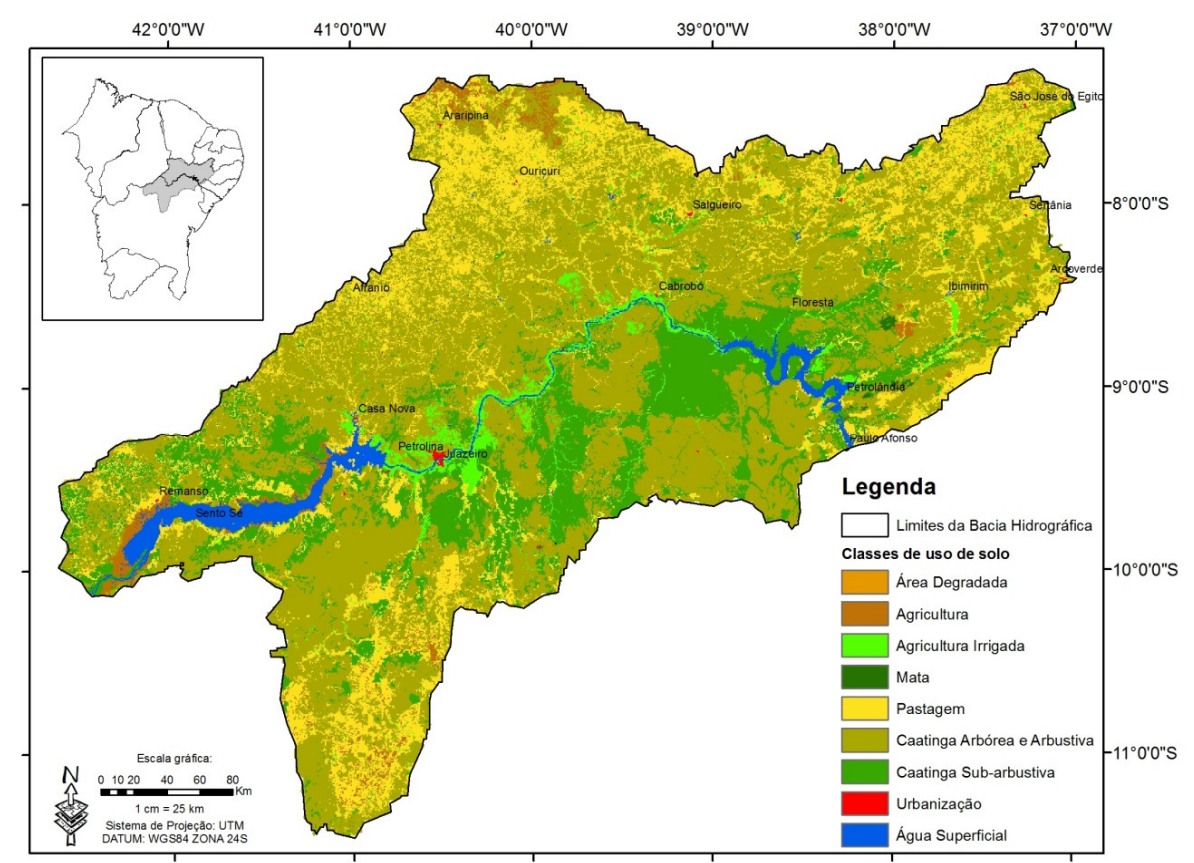

Figura 1. Localização da Bacia Hidrográfica do Submédio Rio São Francisco (BHSRSF). Fonte: Autores (2017). 
A bacia hidrográfica do Submédio Rio São Francisco apresenta predomínio de três classes de solos, sendo mais de $26.463 \mathrm{~km}^{2}$ ou $24 \%$ do total da área da bacia pertencentes a classe de Luvissolo Crômico, a classe de Argilossolo VermelhoAmarelo representa pouco mais de $13.000 \mathrm{~km}^{2}$, a última classe de solo é a do Neossolo Quartzarênico apresentando $11,1 \%$ da área total (Silva et al., 2014).

\section{Dados climáticos}

As simulações realizadas neste estudo para avaliar o impacto da mudança na cobertura do solo sobre o aporte de sedimentos nas condições atuais de uso de solo foram realizadas para o período de 1993 a 2010.

Fizeram parte da composição do banco de dados climático valores diários de precipitação, temperaturas máxima e mínima, radiação solar, velocidade do vento e umidade relativa. Esses dados foram obtidos nas estações meteorológicas de Bebedouro, Cabrobó, Custódia, Ingazeira, Morro do Chapéu, Ouricuri, Petrolândia, Remanso e Verdejante, todas distribuídas na área de estudo (Figura 2). Esses dados são do período de 1970 a 2010 e pertencem às estações da rede de monitoramento meteorológico do Instituto Nacional de Meteorologia (INMET).

\section{Parâmetros do solo}

Foram realizadas coletas de amostras de solo na área de estudo para validar as informações referentes ao mapa de solo. Em seguida, as análises foram efetuadas no Laboratório de Solos da Universidade Federal de Campina Grande. A inserção dos planos de informação cartográficos e dos dados alfanuméricos no modelo ArcSWAT ocorreu via interface apropriada (SIG).

O banco de dados do modelo SWAT possibilita a inserção de novas informações referentes às características dos solos da bacia, dessa forma foi possível adicionar as classes de solos presentes na Bacia Hidrográfica do Submédio Rio São Francisco (BHSRSF) e seus respectivos parâmetros que foram obtidos após a coleta de amostras de solos na área de estudo (Figura 3) e as análises foram efetuadas no Laboratório de Solos da Universidade Federal de Campina Grande. O banco de dados pedológico requer informações das características físico-hídricas de cada classe de solo (Tabela 1), como:

- Grupos de saturação ou hidrológico (HYDGRP);

- Profundidade máxima de solo que a raiz alcança (SOL_ZMX);

- Porosidade (ANION_EXCL);

- Profundidade da camada (SOL_Z);

- Densidade aparente (SOL_BD);

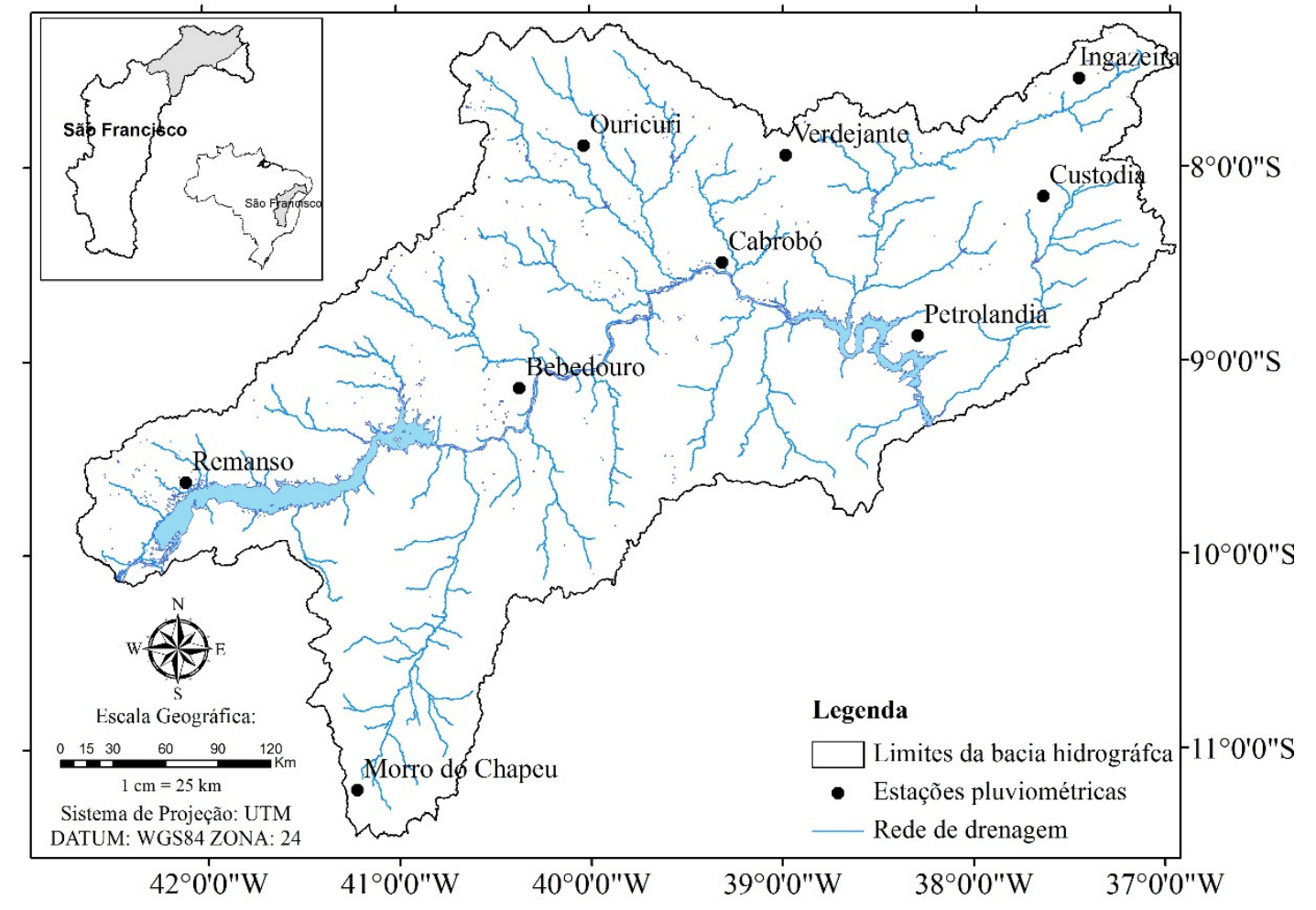

Figura 2. Distribuição das estações meteorológicas do Instituto Nacional de Meteorologia (INMET). Fonte: Autores (2017). 
- Capacidade de água disponível no solo (SOL_AWC);

- Condutividade hidráulica saturada (SOL_K);

- Erodibilidade (USLE_K);

- Albedo, sendo este relativo à parcela da radiação solar que é refletida ao atingir a superfície do solo; considerando sua cobertura vegetal (SOL_ALB);

- Conteúdo de carbono orgânico (SOL_CBN), e Percentagem de argila; silte; areia e rocha.

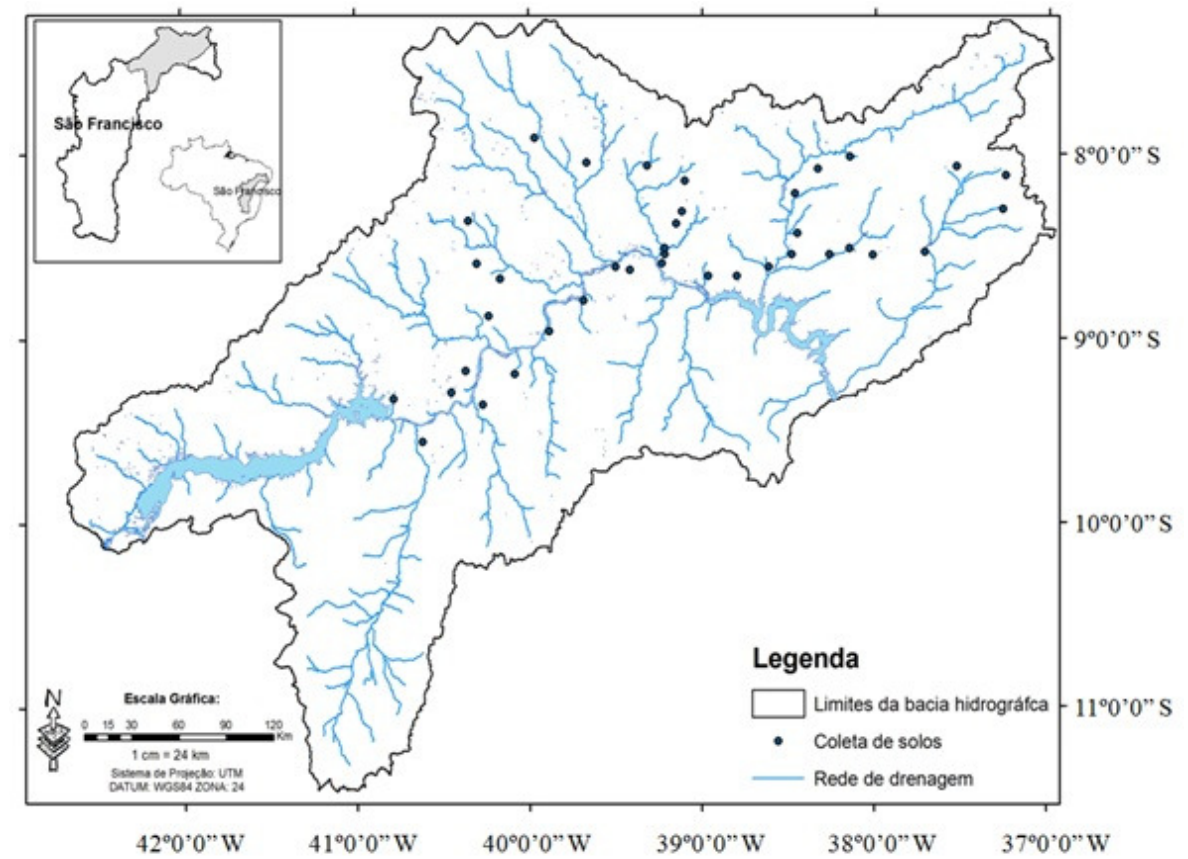

Figura 3. Distribuição dos pontos de obtenção das amostras de solos na Bacia Hidrográfica do Submédio Rio São Francisco (BHSRSF). Fonte: Autores (2017).

Tabela 1. Características físico-hídricas médias dos solos na Bacia Hidrográfica do Submédio Rio São Francisco (BHSRSF). Fonte: Autores (2017).

\begin{tabular}{|c|c|c|c|c|c|c|c|c|c|c|c|c|c|c|}
\hline SNAM & 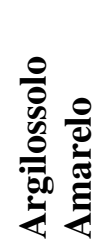 & 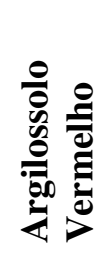 & 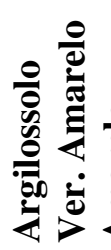 & 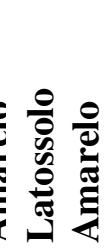 & 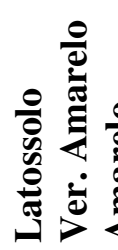 & 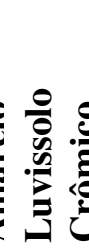 & 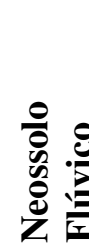 & $\begin{array}{l}0 \\
0 \\
0 \\
0 \\
0 \\
0\end{array}$ & 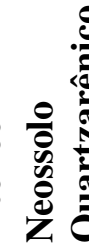 & 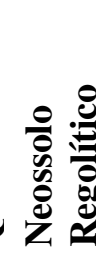 & 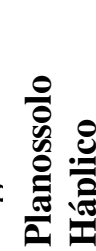 & 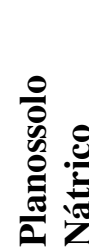 & 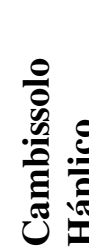 & 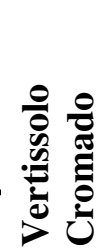 \\
\hline LAYERS & 2 & 2 & 2 & 2 & 2 & 2 & 2 & 2 & 2 & 2 & 2 & 2 & 2 & 2 \\
\hline HYDC & B & B & B & B & & B & & B & A & A & B & & & $\mathrm{C}$ \\
\hline SOL_Z & 600 & 400 & 400 & 400 & 400 & 600 & 400 & 400 & 400 & 400 & 600 & 400 & 600 & 400 \\
\hline $\begin{array}{l}\text { ANION_EX } \\
\text { CL }\end{array}$ & 0,42 & 0,45 & 0,41 & 0,45 & 0,45 & 0,49 & 0,42 & 0,41 & 0,42 & 0,5 & 0,48 & 0,46 & 0,5 & 0,5 \\
\hline SOL_CRK & 0,5 & 0,5 & 0,5 & 0,5 & 0,5 & 0,5 & 0,5 & 0,5 & 0,5 & 0,5 & 0,5 & 0,5 & 0,5 & 0,5 \\
\hline SOL_Z & 200 & 200 & 200 & 200 & 200 & 200 & 200 & 200 & 200 & 200 & 200 & 200 & 200 & 200 \\
\hline SOL_BD & 1,59 & 1,59 & 1,66 & 1,5 & 1,54 & 1,38 & 1,52 & 1,62 & 1 , & 1,55 & 1,43 & 1,46 & 1,37 & 1,52 \\
\hline SOL_A & 0,06 & 0,16 & 0,05 & 0,1 & 0,03 & 0,14 & 0,11 & 0,1 & 0,07 & 0,06 & 0,13 & 0,12 & 0,13 & 0,12 \\
\hline SOL_K & 5,7 & 5,7 & 5,7 & 12,5 & 2,2 & 5,7 & 2,5 & 5,6 & 79,1 & 56,6 & 10,8 & 10,8 & 6,7 & 2,2 \\
\hline SOL_CBN & 1,3 & 1,3 & 1,1 & 1,1 & 6,9 & 1,1 & 5,93 & 0,84 & 0,43 & 0,39 & 0,7 & 0,7 & 1,15 & 5,03 \\
\hline CLAYY & 10 & 13 & 5 & 13 & 10 & 19 & 11 & 3 & 2 & 5 & 17 & 16 & 26 & 56 \\
\hline & 15 & 19 & 12 & 8 & 4 & 2 & 16 & 13 & 1 & 24 & 22 & 14 & 55 & 40 \\
\hline & 75 & 68 & 84 & 79 & 86 & 54 & 73 & 84 & 97 & 71 & 61 & 70 & 19 & 4 \\
\hline ROCK & 0 & 0 & 0 & 0 & 0 & 0 & 0 & 0 & 0 & 0 & 0 & 0 & 0 & 0 \\
\hline SOL_ALB & 0,17 & 0,17 & 0,17 & 0,17 & 0,17 & 0,17 & 0,17 & 0,17 & 0,17 & 0,17 & 0,17 & 0,17 & 0,17 & 0,17 \\
\hline USLE_K & 0,14 & 0,14 & 0,14 & 0,13 & 0,12 & 0,15 & 0,16 & 0,17 & 0,14 & 0,18 & 0,18 & 0,18 & 0,16 & 0,13 \\
\hline
\end{tabular}


Descrição da equação de produção de sedimentos

A erosão do solo foi estimada no modelo SWAT com a Equação Universal de Perda de Solo Modificada (MEUPS). A MEUPS é a versão modificada da Equação Universal de Perda de Solo (EUPS). Essa equação começou a ser implantada no final da década de 1950 pelo Serviço de Conservação dos Solos dos Estados Unidos. É um modelo empírico, baseado em grandes bases de dados de campo, que estima a erosão distribuída e concentrada com base nos valores de quatro grandes fatores intervenientes no processo erosivo: erosividade climática, erodibilidade dos solos, topografia, e uso e manejo da terra (Ward \& Elliot, 1995). O fator de energia da chuva considerado na EUPS é substituído pelo fator de escoamento superficial na MEUPS. Essa modificação permite estimar a produção de sedimentos em eventos individuais. A estimativa da produção de sedimentos foi permitida porque o escoamento superficial é função das condições de umidade antecedente e esse fator representa a energia utilizada pela desagregação e transporte de sedimentos. A Equação Universal de Perda de Solo Modificada segundo (Williams, 1995) foi definida pela Equação (1):

$$
\text { sed }=11,8\left(Q_{\text {sup }} \times q_{\text {pico }} \times \text { Área } a_{H R U}\right)^{0,56} \times K \times C \times L S \times P \times C F R G
$$

onde: $\operatorname{sed}=$ produção de sedimentos após vento de precipitação no dia, em toneladas; $\mathrm{Q}_{\text {sup }}=$ escoamento superficial $(\mathrm{mm}) ; \mathrm{q}_{\text {pico }}=$ vazão de pico do escoamento $\left(\mathrm{m}^{3} \cdot \mathrm{s}^{-1}\right)$; Área $\mathrm{HRU}_{\mathrm{HR}}=$ área da Unidade de Resposta Hidrológica (ha); $\mathrm{K}=$ erodibilidade do solo $\left[\left(0,013\right.\right.$ ton $\left.\mathrm{m}^{2} \mathrm{hr}\right) /\left(\mathrm{m}^{3}\right.$ ton $\left.\left.\mathrm{cm}\right)\right] ; \mathrm{C}=$ é o fator de uso e manejo do solo (adimensional); $\mathrm{LS}=$ fator topográfico (adimensional); $\mathrm{P}=$ fator das práticas conservacionistas (adimensional) e CFRG $=$ fator de fragmentação esparsa (adimensional).

\section{Resultados}

As classes de solos apresentaram contribuições significativas na produção de sedimentos, pois, a partir da distribuição espacial das classes de solos, com suas respectivas predominâncias em cada região da bacia, verificouse que o coeficiente de erodibilidade (fator K) da MUSLE apresentou valores distintos, ou seja, o risco de degradação dos solos apresentou níveis de vulnerabilidade classificados de baixo até alto, segundo a metodologia de Crepani et al. (2001). Tal fator ainda está condicionado aos atributos intrínsecos de cada solo como a suscetibilidade a desagregação e ao transporte das micro partículas de silte e argila, além da sua capacidade de infiltração em função da distribuição dos macro e micro poros. Desta forma, têm-se produção de sedimentos elevadas nas sub-bacias com a presença de solos Neossolo Regolítico e Neossolo Litólico, todavia as sub-bacias com presença de Latossolo Vermelho-Amarelo e Latossolo Amarelo são menos susceptíveis aos processos erosivos.

Ao avaliar a Figura 4, percebe-se que a subbacia em que se insere a estação meteorológica de Remanso localizada na parte oeste da bacia apresentou os maiores picos de produção mensal de sedimentos para o ano de 2010 e no cenário atual de uso de solo, as maiores concentrações são verificadas entre os meses de janeiro a abril. Os valores médios são de aproximadamente 35 t.ha ${ }^{1}$. mês $^{-1}$. Para essa sub-bacia, destaca-se que o mês de dezembro de 2010 apresentou registros superiores a 50 t.ha $^{-1} \cdot \mathrm{mês}^{-1}$. Tal característica na variabilidade do aporte de sedimentos pode estar associada às configurações na cobertura do solo da bacia associado ao regime da precipitação na região. Entretanto o maior pico de aporte de sedimentos foi verificado para sub-bacia de Bebedouro localizada na parte centro-oeste da bacia, foi registrado valor superior a 60 t.ha ${ }^{-1} \cdot \mathrm{mês}^{-1}$ para o mês de abril de 2010. A sub-bacia destaca-

se pela maior presença de áreas irrigadas nas margens do rio São Francisco.

A produção de sedimentos obtida neste trabalho é semelhante à encontrada por Machado (2002) que utilizou o SWAT na bacia do rio Marins em São Paulo, que possui uma área de 5,973 ha, e uso dominante de cana-de-açúcar e pastagem, tendo produção média de sedimento entre 1999 e 2000 de 16,935 t.ha' ${ }^{-1}$. Grossi (2003), na bacia do rio Pardo em Botucatu-SP, com área de 1.291,64 ha e uso predominantemente agrícola e pastagem, obteve uma produção de sedimentos média para o período entre 1997 e 2001 de 44,01 t.ha ${ }^{-1}$.

Os menores registros de aporte de sedimento foram observados para sub-bacia de Petrolândia, na qual a predomínio de vegetação nativa do tipo arbórea e sub-arbustiva. O fator de cobertura atua como proteção natural da superfície, mesmo que pouco densa, tem efeito significativo para redução na degradação do solo, pode-se associar ainda que a região apresente pouca declividade, e as condições climáticas da região semiárida geram pouco escoamento superficial. O maior pico de produção foi visualizado no mês de dezembro, com registros próximos de 30 t.ha ${ }^{-1} \cdot \mathrm{mês}^{-1}$. 


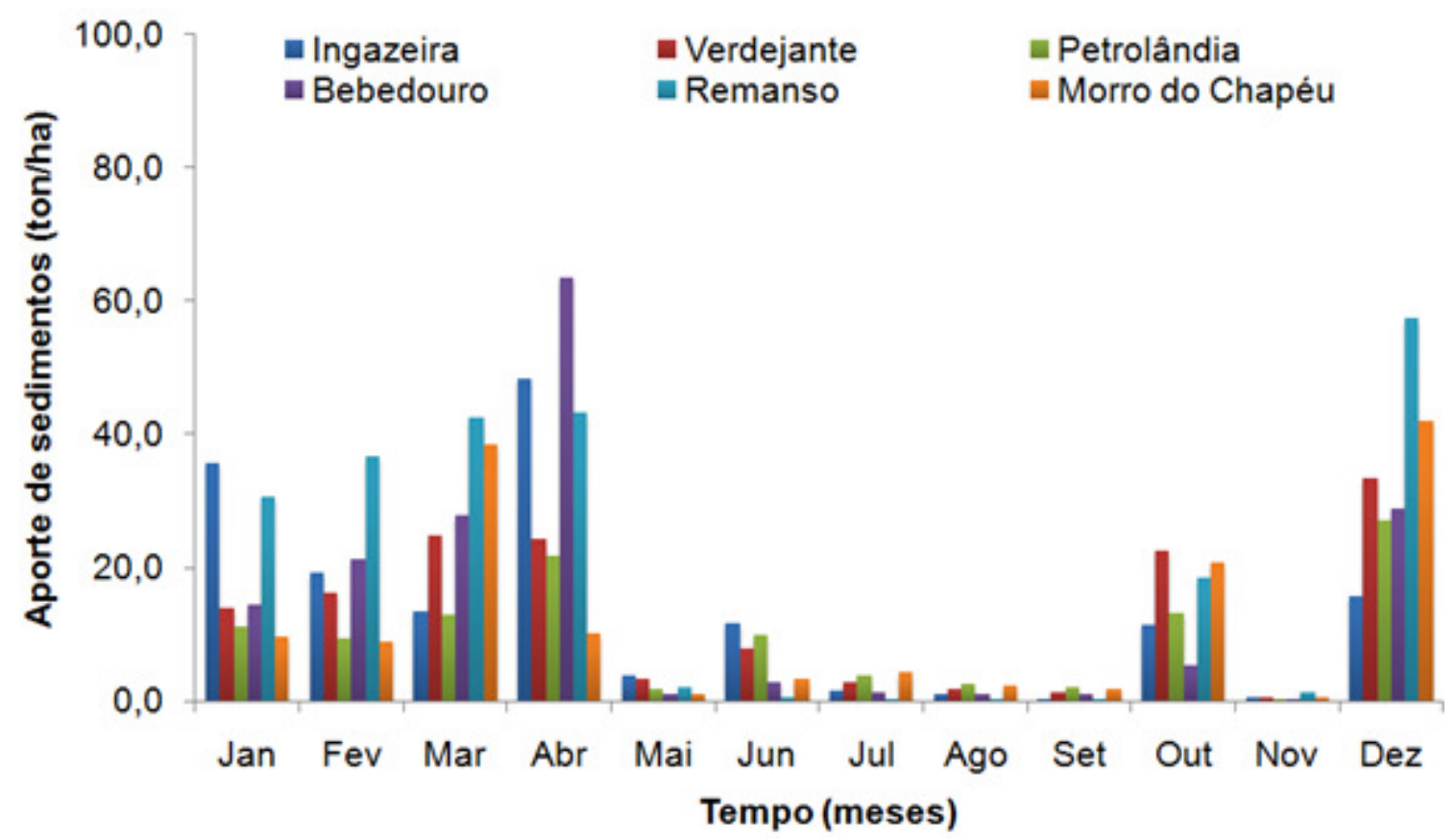

Figura 4. Variabilidade mensal do aporte de sedimentos para o cenário atual de uso e cobertura do solo no de ano de 2010. Fonte: Autores (2017).

\section{Discussão}

A relação entre totais pluviométricos e produção de sedimentos apresentada neste trabalho, foi encontrada por Machado (2002) na bacia do ribeirão Marins, em Piracicaba, utilizando o SWAT, constatando que $94 \%$ dos sedimentos produzidos estavam associados ao período chuvoso. Tal relação também foi encontrada por Behera et al. (2006), em uma bacia situada no Distrito de Mindinapore, no oeste de Bengal, na Índia; por He et al. (2008) no rio Hei, na província de Shaanki, na China; por Zhang et al. (2007) na porção superior do rio Amarelo, na China.

Em muitos solos, as partículas de silte e argila desprendidas no processo erosivo formam uma camada compactada (superfície de selamento) contribuindo para a redução da taxa de infiltração da água no solo (Chaves et al., 1993). Segundo Reichert et al. (1992) a textura do solo afeta sensivelmente o salpico de partículas com o impacto das gotas de chuva, o que contribui para uma redução da porosidade da camada superficial do solo. Além do impacto da gota, Morin \& van
Winkel (1996) citam a dispersão físico-química das argilas do solo como causas da formação do selamento superficial e, consequentemente, da redução da velocidade de infiltração. Por outro lado, a manutenção de resíduos culturais sobre a superfície do solo, elevando a sua rugosidade, associada à pequena movimentação mecânica do solo, favorece um controle mais eficaz da erosão hídrica. Segundo Leite et al. (2002), os preparos conservacionistas possibilitam, de modo geral, maior retenção dos resíduos, promovendo aumento da tensão crítica de cisalhamento e, em consequência, a resistência do solo à erosão hídrica.

Analisando as condições médias mensais das componentes do balanço hidrossedimentológico para o ano de 2010 nas condições atuais de acordo com a Tabela 2 observa-se que grande parte da precipitação é removida principalmente por meio da evapotranspiração ETr $(61,1 \%)$, a segunda parcela mais significativa é atribuída ao escoamento superficial com cerca de $23,4 \%$.

Tabela 2. Médias mensais das componentes do balanço hidrossedimentológico para o ano de 2010 nas condições atuais. Fonte: Autores (2017).

\begin{tabular}{|c|c|c|c|c|c|c|c|}
\hline \multirow{2}{*}{ Mês } & Precipitação & Runoff & $\begin{array}{l}\text { Fluxo } \\
\text { lateral }\end{array}$ & ETr & ETP & $\begin{array}{c}\text { Produção } \\
\text { de água }\end{array}$ & \multirow{2}{*}{$\begin{array}{c}\text { Aporte de } \\
\text { sedimentos } \\
\left.\text { (t.hat }^{-1}\right)\end{array}$} \\
\hline & \multicolumn{6}{|c|}{$(\mathbf{m m})$} & \\
\hline Janeiro & 94,3 & 17,7 & 0,9 & 43,5 & 182,5 & 0,88 & 20,5 \\
\hline Fevereiro & 83,5 & 13,6 & 0,06 & 29,3 & 159,8 & 0,96 & 18,8 \\
\hline Março & 116,5 & 31,4 & 0,62 & 88,0 & 180,4 & 12,4 & 29,3 \\
\hline Abril & 136,3 & 45,6 & 0,86 & 83,7 & 147,5 & 29,7 & 37,1 \\
\hline
\end{tabular}




\begin{tabular}{lccccccc} 
Maio & 11,5 & 0,5 & 0,15 & 56,3 & 136,8 & 10,1 & 1,8 \\
Junho & 30,2 & 2,2 & 0,05 & 18,1 & 111,0 & 4,2 & 6,2 \\
Julho & 11,8 & 0,6 & 0,03 & 13,4 & 115,3 & 1,0 & 2,0 \\
Agosto & 7,5 & 0,4 & 0,03 & 21,0 & 144,7 & 0,4 & 1,2 \\
Setembro & 6,9 & 0,4 & 0,02 & 11,6 & 162,2 & 0,2 & 1,2 \\
Outubro & 57,3 & 6,5 & 0,02 & 11,7 & 194,6 & 0,1 & 12,8 \\
Novembro & 4,7 & 0,3 & 0,02 & 18,2 & 168,9 & 0,1 & 0,4 \\
Dezembro & 119,3 & 40,2 & 0,02 & 20,6 & 179,9 & 0,1 & 34,1 \\
\hline
\end{tabular}

O complemento das componentes do balanço hídrico é associado aos processos de manutenção da umidade do solo, infiltração e percolação / recarga das águas subterrâneas $(15,5 \%)$. Destaca-se ainda que a produção de água ou taxa de entrega de água aos canais da rede de drenagem da bacia hidrográfica representa está relacionada ao escoamento superficial, que foi a principal componente da geração das vazões observadas nas simulações com modelo SWAT. Isto pode ser atribuído a uma limitação da recarga de água subterrânea da região semiárida do nordeste do Brasil, devido principalmente as características geológicas/edáficas no que se refere a genesis da formação dos solos, tornando-os pouco profundos, com a presença de afloramentos rochosos na paisagem sertaneja, outra característica é associada impermeabilidade dos solos devido ao seu alto teor de argila e silte.

Segundo Tucci (1998) em bacias rurais das regiões Sul e Sudeste do Brasil, a precipitação é geralmente da ordem de $1.500 \mathrm{~mm}$. Deste total, estima-se que $68 \%$ é evapotranspiração, de 6,4 a $16 \%$ é escoamento superficial e de 16 a $25,4 \%$ refere-se ao escoamento subterrâneo.

\section{Conclusão}

O modelo SWAT permitiu a análise dos componentes do balanço hidrossedimentológico em regiões semiáridas, cujo desempenho pode ser considerado satisfatório, ou seja, os resultados mostram sua aplicabilidade para diferentes condições hidrológicas e de uso do solo na região semiárida do nordeste brasileiro.

A exposição do solo ao processo erosivo gera alterações em sua qualidade, comprometendo a rentabilidade das atividades econômicas desenvolvidas na região da bacia.

O gerenciamento da paisagem em bacias hidrográficas através do modelo SWAT pode ajudar na identificação de áreas que necessitam de práticas de controle dos processos erosivos.

\section{Agradecimentos}

Os autores agradecem à Universidade Federal de Campina Grande-UFCG pelo apoio técnico e científico.

\section{Referências}

ARAGÃO, R.; CRUZ, M. A. S.; AMORIM, J. R. A.; MENDONÇA, L. C.; FIGUEIREDO, E. E.; SRINIVASAN, S. 2013. Análise de sensibilidade dos parâmetros do modelo SWAT e simulação dos processos hidrossedimentológicos em uma bacia no agreste nordestino. Revista Brasileira de Ciência do Solo, v. 37, p. 1091-1102.

BEHERA S, PANDA R. 2006. Evaluation of management alternatives for an agricultural watershed in a sub-humid subtropical region using a physical process based model. Agriculture, Ecosystems and Environment, v. 113, p. 62-72.

CHAVES, H. M. L.; ORLOWSKI, E.; ROLOFF, G. 1993. Previsão da infiltração sob condições dinâmicas de selamento superficial. Revista Brasileira de Ciência do Solo, v. 17, n. 2, p. 141147.

CIBIN, R.; SUDHEER, K. P.; CHAUBEY, I. 2010. Sensitivity and identifiability of stream flow generation parameters of the SWAT model. Hydrological Processes, v. 24, p. 1133-1148.

CODEVASF - Companhia de Desenvolvimento dos Vales do São Francisco e do Parnaíba. 1999. Inventário de projetos. Brasília: rev. atual. 3. ed. 223p.

CREPANI, E.; MEDEIROS, J. S. DE; AZEVEDO, L. G. DE; HERNANDEZ FILHO, P.; FLORENZANO, T. G.; DUARTE, V. 2001. Curso de sensoriamento remoto aplicado ao zoneamento ecológico-econômico. São José dos Campos: INPE. 25p.

FUKUNAGA, D. C.; CECÍLIO, R. V.; ZANETTI, S. S.; OLIVEIRA, L. T.; CAIADO, M. A. C. 2015. Application of the SWAT hydrologic model to a 
tropical watershed at Brazil. Catena, v. 125, p. 206213.

GALHARTE, C. A.; VILLELA, J. M.; CRESTANA, S. 2014. Estimativa da produção de sedimentos em função da mudança de uso e cobertura do solo. Revista Brasileira de Engenharia Agrícola e Ambiental, v. 18, p. 194-201.

GROSSI, C. H. 2003. Sistema e informação geográfica - Basins 3.0 na modelagem hidrológica da Bacia Experimental do Rio Pardo, SP. 101f. Dissertação (Mestrado em Agronomia), Faculdade de Ciências Agronômicas, Universidade Estadual Paulista, Rio Pardo.

HE, Q.; WALLING, D. E. 1996. Use of fallout Pb210 measurements to investigate longer-term rates and patterns of overbank sediment deposition on the floodplains of lowland rivers. Earth Surface Processes and Landforms, v. 21, n. 2, 141-154.

HUNDECHA, Y. H.; OUARDA, T. B. M. J.; BÁRDOSSY, A. 2008. Regional estimation of parameters of a rainfall-runoff model at ungauged watersheds using the "spatial" structures of the parameters within a canonical physiographicclimatic space. Water Resources Research, v. 44, p. 1427-1440.

KIM, N. W.; LEE, J. 2010. Enhancement of channel routing module in SWAT. Hydrological Processes, v. 24, p. 96-107.

LEITE, D.; BERTOL, I.; ZAPORALLI, AS.; CARRAFA, R. 2002. Erosão hídrica sob chuva simulada no milho. In: Reunião Brasileira de Manejo e Conservação do Solo e da Água, 14, Cuiabá. Anais...Cuiabá: Sociedade Brasileira de Ciência do Solo. CD-Rom

MACHADO, R. E.; VETTORAZZI, C. A. 2003. Simulação da produção de sedimentos para a microbacia hidrográfica do ribeirão dos Marins, SP. Revista Brasileira de Ciências do Solo, v. 4, p. 735-741.

MORIN, J.; VAN WINKEL, J. 1996. The effect of raindrop impact and sheet erosion on infiltration rate and crust formation. Soil Science Society of America Journal, v. 60, p. 1223-1227.

OEURNG, C.; SAUVAGE, S.; SANCHEZ PEREZ, J. M. 2011. Assessment of hydrology, sediment and particulate organic carbon yield in a large agricultural catchment using the SWAT model. Journal of Hydrology, v. 401, p. 145-153.
PEREIRA, D. R.; ALMEIDA, A. Q.; MARTINEZ, M. A.; ROSA, D. R. Q. 1024a. Impacts of deforestation on water balance components of a watershed on the Brazilian east coast. Revista Brasileira de Ciência do Solo, v. 38, p. 1350-1358, 2014a.

PEREIRA, D. R.; MARTINEZ, M. A.; ALMEIDA, A. Q.; PRUSKI, F. F.; SILVA, D. D.; ZONTA, J. H. 2014b. Hydrological simulation using swat model in headwater basin in southeast Brazil. Engenharia Agrícola, v. 34, n. 4, p. 789799.

REICHERT, J. M.; VEIGA, M; CABEDA, M. S. V. 1992. Selamento superficial e infiltração de água em solos do Rio Grande do Sul. Revista Brasileira de Ciência do Solo, v.16, n.3, p.289-298.

SETEGN, S. G.; SRINIVASAN, R.; MELESSE, A. M.; DARGAHI, B. 2010. SWAT model application and prediction uncertainty analysis in the Lake Tana Basin, Ethiopia. Hydrological Processes, v. 24, n. 3, p.357-367.

SILVA, M. T.; SILVA, V. P. R.; SOUZA, E. P.; ARAÚJO, A. L. 2015. SWAT model application to estimate the flow in the basin of lower-middle São Francisco River. Revista Brasileira de Geografia Física, v. 8, p. 1615-1627.

SILVA, M. T.; SILVA, V. P. R.; SOUZA, E. P.; ARAÚJO, A. L.; AZEVEDO, J. V. V. 2016a. Estimativa da Erosividade das Chuvas na Estação Agrometeorológica de Bebedouro (PE). In: SANTOS, C. A. C. DOS; SILVA, M. T.; SILVA, V. M. DE A. (Org.). Recursos Naturais do Semiárido - Estudos Aplicados. 1 ed. Campina Grande-PB, v. 1, pp. 325-339.

SILVA, M. T.; SILVA, V. P. R.; SOUZA, E. P.; OLIVEIRA, V. G. 2014. Morphometric analysis of the basin low middle São Francisco River. Journal of Hyperspectral Remote Sensing, v. 4, n. 8, p. 168174.

SILVA, V. P. R.; SILVA, M. T.; SOUZA, E. P. 2016b. Influence of land use change on sediment yield: a case study of the sub-middle of the São Francisco River basin. Engenharia Agrícola, v. 36, n. 6, p. 1005-1015.

TUCCI, C. E. M.; CLARKE, R. T. 1998. Environmental Issues in the La Plata Basin. Water Resources Development, v. 14, n. 2, p. 157-173. 
Journal of Environmental Analysis and Progress V. 02 N. 03 (2017) 203-211

WARD, A. D.; ELLIOT, W. J. 1995. Environmental Hidrology. New York: Lewis Publishers. 328p.

WILLIAMS, J. R. 1995. The EPIC model. In: Singh, V. P. Computer models of watershed hydrology. Highlands Ranch: Water Resources Publications. pp. 909-1000.
ZHANG, H. G.; FU, S. H.; FANG, W. H.; IMURA, H.; ZHANG, X. C. 2007. Potential effects of climate change on runoff in the Yellow River Basin of China. Transactions of the ASABE, v. 50, p. 911-918. 